\title{
SPECIAL RELATIVITY AT ACTION IN THE UNIVERSE
}

\author{
Gabriele Ghisellini \\ Osservatorio Astronomico di Brera, v. Bianchi 46, I-23807 Merate, Italy \\ e-mail address: gabriele@merate.mi.astro.it
}

\begin{abstract}
Nature succeeds in accelerating extended and massive objects to relativistic velocities. Jets in Active Galactic Nuclei and in galactic superluminal sources and gamma-ray bursts fireballs have bulk Lorentz factors from a few to several hundreds. A variety of effects then arises, such as the beaming of the produced radiation, light aberration, time contraction and the Doppler frequency shift. I will emphasize that special relativity applied to real (i.e. extended) observed objects inevitably must take into account that any piece of information is carried by photons. Being created in different parts of the source, they travel different paths to reach the observer, depending on the viewing angle. The object is seen rotated, not contracted, and at small viewing angles time intervals are observed shorter than intrinsic ones.
\end{abstract}

\section{Introduction}

We are used to consider earth's particle accelerators as the realm of special relativity. But nature can do better and bigger, and is able to accelerate really large masses to relativistic speeds. Besides cosmic rays, we now know that in the jets of active galactic nuclei (AGN) which are also strong radio emitters plasma flows at $v \sim 0.99 c$, and that in gamma-ray bursts (GRB) the fireball resulting from the release of $\sim 10^{52} \mathrm{erg}$ in a volume of radius comparable to the Schwarzschild radius of a solar mass black hole reaches $v \sim 0.999 c$. These speeds are so close to the light speed that it is more convenient to use the corresponding Lorentz factor of the bulk motion, $\Gamma=\left(1-\beta^{2}\right)^{-1 / 2}$ : for $\Gamma \gg 1$, we have $\beta \sim 1-1 /\left(2 \Gamma^{2}\right)$.

The energetics involved is huge. In AGNs, a fraction of a solar mass per year can be accelerated to $\Gamma \sim 10$, leading to powers of $\sim 10^{46} \mathrm{erg} \mathrm{s}^{-1}$ in bulk motion. In GRBs, the radiation we see, if isotropically emitted, can reach $10^{54} \mathrm{erg} \mathrm{s}^{-1}$, suggesting even larger values for the bulk motion power. Recently, very interesting sources have been discovered within our Galaxy through their activity in X-rays: they occasionally produce radio jets closely resembling those of radio-loud quasars. During these ejection episodes, the power in bulk motion can exceed $10^{40} \mathrm{erg} \mathrm{s}^{-1}$, a value thought to exceed the Eddington limit for these sources.

The study of these extended objects moving close to $c$ requires to take into account the different travel paths of the photons reaching us. Curiously enough, the resulting effects had not been studied until 1959, when Terrel (1959) pointed out that a moving sphere does not appear contracted, but rotated, contrary to what was generally thought (even by Einstein himself ...). These results, which were "academic" in those years, are now fully applied to the above mentioned relativistic cosmic objects.

In this paper I will present some of the evidence in support of relativistic bulk motion in astronomical objects, and then discuss how "text-book special relativity" has to be applied when information are carried by photons.

\section{Superluminal motion}

Rees (1966) realized that an efficient way of transporting energy from the vicinity of super-massive black holes to the radio lobes of the recently discovered radiogalaxies is through the bulk motion 


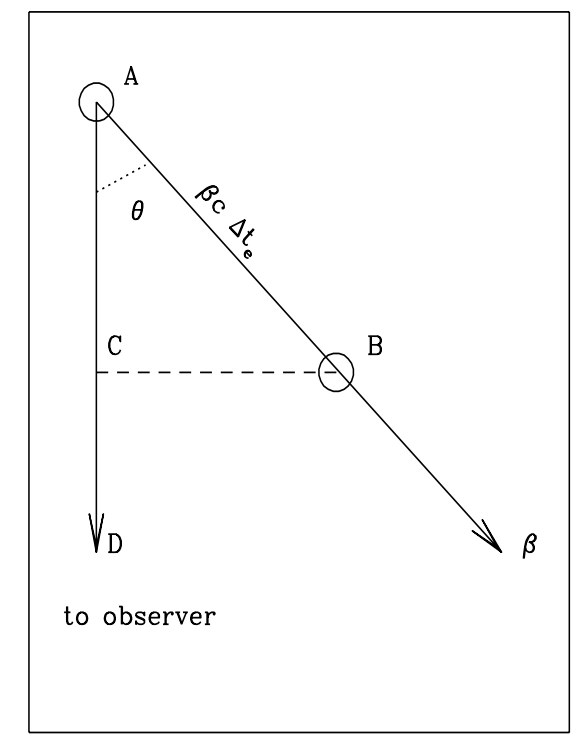

Figure 1: Explanation of the apparent superluminal speed. A blob emits a photon from $A$ and, after a time $\Delta t_{e}$, from $B$. If the true velocity is close to the speed of light and the angle $\theta$ is sufficiently small, the apparent velocity will exceed the speed of light.

of relativistically moving plasma. If this plasma emits radiation on its way, then we ought to see moving spots of emission in radio maps. One of the most spectacular prediction by Rees was that this motion could appear to exceed the speed of light. This was indeed confirmed in the early seventies, when the radio-interferometric techniques allowed to link radio telescopes thousands of kilometers apart. Among the first few observed targets was 3C 279, a radio-loud quasar at a redshift of $z=0.538$. Bright spots in radio maps taken at interval of months were apparently moving at a speed exceeding 10 times $c$. For obvious reasons, sources presenting this phenomenon are referred to as superluminal sources.

This phenomenon can be simply explained, as long as the plasma is moving at velocities close to $c$ at small viewing angle (i.e. the angle between the velocity vector and the observer's line of sight). Consider Fig. 1: suppose the moving blob emits a photon from positions $A$ and then from $B$. The time between the two emissions, as measured by an observer which sees the blob moving, is $\Delta t_{e}$. Therefore the distance $A B$ is equal to $\beta c \Delta t_{e}$, and $A C=\beta c \Delta t_{e} \cos \theta$. In the same time interval, the photon emitted in $A$ has reached the point $D$, and the distance $A D$ is equal to $c \Delta t_{e}$. Thus the two photons are now separated by $D C=A D-A C=c \Delta t_{e}(1-\beta \cos \theta)$. The difference between the arrival times of these two photons is then $\Delta t_{a}=\Delta t_{e}(1-\beta \cos \theta)$, and the projected separation of the blob in the two images is $C B=c \beta \Delta t_{e} \sin \theta$, leading to an apparent velocity:

$$
\beta_{\text {app }}=\frac{\beta \sin \theta}{1-\beta \cos \theta}
$$

It can be readily seen that $\beta_{a p p}>1$ for $\beta \rightarrow 1$ and small viewing angles $\theta$. The apparent speed is maximized for $\cos \theta=\beta$, where $\beta_{a p p}=\beta \Gamma$. Notice that this simple derivation does not require any Lorentz transformation (no $\Gamma$ factor involved!). The superluminal effect arises only from the Doppler contraction of the arrival times of photons. 


\section{Beaming}

Let us assume that a source emits isotropically in its rest frame $K^{\prime}$. In the frame $K$, where the source is moving relativistically, the radiation is strongly anisotropic, and three effects occur:

- Light aberration: photons emitted at right angles with respect to the velocity vector (in $K^{\prime}$ ) are observed in $K$ to make an angle given by $\sin \theta=1 / \Gamma$. This means that in $K$ half of the photons are concentrated in a cone of semi-aperture angle corresponding to $\sin \theta=1 / \Gamma$.

- Arrival time of the photons: as discussed above, the emission and arrival time intervals are different. As measured in the same frame $K$ we have, as before, $\Delta t_{a}=\Delta t_{e}(1-\beta \cos \theta)$. If $\Delta t_{e}^{\prime}$ is measured in $K^{\prime}, \Delta t_{e}=\Gamma \Delta t_{e}^{\prime}$ leading to

$$
\Delta t_{a}=\Gamma(1-\beta \cos \theta) \Delta t_{e}^{\prime} \equiv \frac{\Delta t_{e}^{\prime}}{\delta}
$$

Here we have introduced the factor $\delta$, referred to as the beaming or Doppler factor. It exceeds unity for small viewing angles, and if so, observed time intervals are contracted.

- Blueshift/Redshift of frequencies: since frequencies are the inverse of times, we just have $\nu=\delta \nu^{\prime}$.

It can be demonstrated (see e.g. Rybicki \& Lightman 1979) that the specific intensity $I(\nu)$ divided by the cube of the frequency is Lorentz invariant, and therefore

$$
I(\nu)=\delta^{3} I^{\prime}\left(\nu^{\prime}\right)=\delta^{3} I^{\prime}(\nu / \delta) .
$$

Integration over frequencies yields $I=\delta^{4} I^{\prime}$. The corresponding transformation between bolometric luminosities sometimes generates confusion. It is often said that $L=\delta^{4} L^{\prime}$. What this means is: if we estimate the observed luminosity $L$ from the received flux under the assumption of isotropy, this is related to $L^{\prime}$ through the above equation.

But suppose that the photon receiver covers the entire sky, i.e. completely surrounds the emitting source: what is then the relation between the received power in the frames $K$ and $K^{\prime}$ ? In this case we must integrate $d L / d \Omega$ over the solid angle obtaining:

$$
L=\int \delta^{4} \frac{L^{\prime}}{4 \pi} 2 \pi \sin \theta d \theta=\Gamma^{2} \frac{\beta^{2}+3}{3} L^{\prime} \rightarrow \frac{4}{3} \Gamma^{2} L^{\prime}
$$

Note that in this situation the power is not a Lorentz invariant. This is because here we are concerned with the power received in the two frames, not with the emitted one (which is indeed Lorentz invariant). This is yet another difference with respect to "text-book" special relativity not accounting for photons: here the time transformation involves the Doppler term $(1-\beta \cos \theta)$, causing the difference between emitted and received power (see also Rybicki \& Lightman 1979, p. 141).

Because of beaming, relativistically moving objects appear much brighter if their beams point at us, and can therefore be visible up to large distances. Besides being extremely important in order to calculate the intrinsic physical parameters of a moving source, beaming is also crucial for the moving object itself. The observed objects are rarely isolated and more often are part of a jet immersed in a bath of radiation. Just for illustration, let us consider a blob moving close to an accretion disk and surrounded by gas clouds responsible for the emission of the broad lines seen in the spectra of quasars: as the blob moves at relativistic speed in a bath of photons it 
will see this radiation enhanced. Furthermore, because of aberration, in its frame most of the photons will appear to come from the hemisphere towards which the blob is moving, and be blueshifted. For an observer at rest with respect to the photon bath, the radiation energy density seen by the blob is enhanced by $\sim \Gamma^{2}$. This increases the rate of interaction between the photons and the electrons in the blob, leading to enhanced inverse Compton emission and possibly even deceleration of the blob by the so called Compton drag effect.

\section{Evidences for relativistic motion}

\subsection{Radio-loud AGN with flat radio spectrum}

- Superluminal motion - The most striking evidence of bulk motion comes from the observation of superluminal sources. Improvements in the interferometric techniques have led to the discovery of more than 100 of these sources (see e.g. Vermeulen \& Cohen 1994). The typical bulk Lorentz factors inferred range between 5 and 20 .

- Compton emission - From radio data (size, flux, spectrum) one can derive, through the synchrotron theory, the number density of emitting particles and the radiation energy density (Hoyle, Burbidge \& Sargent 1966). These quantities determine the probability that particles and photons interact through the inverse Compton process, and thus it is possible to predict the amount of the high energy radiation (i.e. X-rays) produced. However, this estimated flux is often orders of magnitude larger than what is observed, if beaming is not taken into account. Conversely, the requirement that the radio source emits at most the observed $\mathrm{X}$-ray flux, sets a (lower) limit on the beaming factor $\delta$. Typical values are in agreement with those derived from superluminal motion (Ghisellini et al. 1993).

- High brightness temperatures - This argument is similar to that just presented. The brightness temperature $T_{b}$ [defined through $I(\nu) \equiv 2 k T_{b} \nu^{2} / c^{2}$ ] is related to the density of particles and photons, and therefore to the probability of Compton scattering: high brightness temperature implies powerful Compton emission. More precisely, if $T_{b}>10^{12} \mathrm{~K}$ (this value is called the Compton limit), the luminosity produced by the first order Compton scattering is larger than the synchrotron luminosity, and that in the second order exceeds (by the same factor) that in the first order, and so on. Clearly, this can only occur until the typical photon energy reaches the typical electron energy, above which the power has to drop. This increasingly important particle cooling is called the Compton catastrophe. To avoid it, we resort to beaming, recalling that $T_{b}$ transforms according to

$$
\begin{aligned}
& T_{b}=\delta T_{b}^{\prime} \quad \text { Source size measured directly } \\
& T_{b}=\delta^{3} T_{b}^{\prime} \quad \text { Source size measured through variability }
\end{aligned}
$$

The Compton limit of $T_{b}>10^{12} \mathrm{~K}$ is derived by requiring that the radiation energy density is smaller than the magnetic energy density. A more severe limit can be obtained imposing the condition of equipartition between particle and magnetic energy densities, as proposed by Readhead (1994). In the latter case one derives larger $\delta$ factors, which are in agreement with those obtained by the two previous methods. A note of caution: there is a significant number of sources, called intraday variables, whose radio flux changes on a timescale of hours. For them, $T_{b}>10^{18} \mathrm{~K}$, and therefore it is inferred $\delta>100$, a value too large to be consistent with the those derived in other ways. This is an open issue, and probably effects due to interstellar scintillation and/or coherent radiation (as in pulsars) have to been invoked (for a review see Wagner \& Witzel 1995). 
- Gamma-ray emission - The $\gamma$-ray satellite CGRO discovered that radio-loud quasars with flat radio spectrum (FSRQ) can be strong $\gamma$-ray emitters, and that often most of their power is radiated in this band. This emission is also strongly variable, on timescales of days or less.

However, photons above $m_{e} c^{2}=511 \mathrm{keV}$ can interact with lower energy photons, producing electron-positron pairs. This happens if the optical depth for the photon-photon interaction is greater than unity, and this depends on the density of the target photons and the typical size of the region they occupy. If not beamed, the large power and rapid variability observed imply optical depths largely in excess of unity, and so $\gamma$-ray would be absorbed within the source. The condition of transparency to this process leads to lower limits on beaming factors somewhat smaller than in the previous cases. There are also objects (still a few, but increasing in number) observed above a few tenths of a $\mathrm{TeV}$ (from the ground by Cherenkov telescopes). The corresponding limits on $\delta$ are the most severe.

- One-sidedness of jets - Radio-sources are very often characterized by two lobes of extended emission, but in some of them only one jet - starting from the nucleus and pointing to one of the lobes - is visible. If the emitting plasma is relativistically moving, the radiation from the jet approaching us is enhanced, while that from the receding jet is dimmed, explaining the observed asymmetry. This is often referred to as Doppler favoritism.

- Super-Eddington luminosities - FSRQs show violent activity: large luminosity changes on short timescales. If the variability timescales are associated to the Schwarzschild radius (hence the black hole mass) luminosities exceeding the Eddington limit are often derived (if isotropy is assumed). This difficulty can be easily overcome by beaming (which affects both the variability timescales and the observed power).

- The Laing-Garrington effect - The two lobes of radio sources are often differently polarized (or, more precisely, they are differently depolarized): as always the (one sided) jet points towards the more polarized lobe, this convincingly indicates that the visible jet is the one approaching the observer (Laing 1988, Garrington et al. 1988).

- Jet bending - Jets of AGNs are often significantly curved, posing problems of stability. But the strong bendings could be largely apparent, if the jet is seen at small viewing angles. This constitutes a further independent hint that these jets are pointing at us (although is not direct evidence of relativistic motion).

- Parent population — For each source whose jet is direct towards us, there must be $\sim \Gamma^{2}$ other sources with jets pointing away. If beaming is important, then these objects must be less luminous, not (extremely) superluminal, and not showing violent activity. This parent population of sources can be identified with that of radiogalaxies (Blandford \& Rees 1978; for a review see Urry \& Padovani 1995): indeed their number agrees with what expected from the beaming parameters derived by the other methods.

\subsection{Galactic superluminal sources}

In 1994, the two X-ray transients GRS1915+105 and J1655-40 were monitored with the VLA during a campaign aimed at finding radio jets associated with galactic sources. Surprisingly, it was discovered superluminal motion in both of them (Mirabel \& Rodriguez 1994; Hjellming \& Rupen 1995). The most interesting observational fact is that in these two sources we see both the jet and the counter-jet. This is unprecedented among superluminal sources, and it is possible because: i) the viewing angle is large, suppressing the Doppler favoritism effect; ii) the bulk 
Lorentz factor is small, $\Gamma=2.5$, leading to a moderate beaming and thus an observable flux even at large viewing angles.

Under the assumption that the superluminal blobs move in opposite directions and are characterized by the same (true) velocity $\beta c$, we can apply Eq. (1) twice to derive both $\beta$ and $\theta$. This in turn determines $\delta$.

Very severe limits can be set on the power carried by the moving blob in the form of both particle bulk motion and Poynting flux. The radio emission observed with VLA comes from a region $\sim 10^{15} \mathrm{~cm}$ in size, and is believed to be produced by synchrotron emission: it is then possible to calculate the number of particles responsible for the observed radiation (this is a lower limit, since other non emitting, i.e. sub relativistic, particles might be present).

The number inferred depends on the value of the magnetic field $B$ : an increase of $B$ decreases the amount of particles required, but increases the implied Poynting flux. The sum of the power in particle bulk motion and in Poynting flux has therefore a minimum. For GRS1915+105, this is of the order of $10^{40} \mathrm{erg} \mathrm{s}^{-1}$ (Gliozzi, Bodo \& Ghisellini, 1999), and exceeds by a factor $\sim 10$ the power emitted by the accretion disk (in soft and medium energy $\mathrm{X}$-rays). This result itself puts strong constraints on any model for the acceleration of jets, by excluding the possibility that this occurs through radiation pressure.

\subsection{Gamma-ray Bursts}

GRBs are flashes of hard $\mathrm{X}$ - and $\gamma$-rays, lasting for a few seconds. Discovered by the Vela satellites in the late sixties, their origin remained mysterious until the Italian-Dutch satellite BeppoSAX succeeded in locating them on the sky with small enough error-boxes: prompt followup observations were then possible and led to the discovery that they are at cosmological distances. This in turn allowed to estimate the energy involved which, if the radiation is emitted isotropically, is in the range $10^{52}-10^{54} \mathrm{erg}$. The energetics is in itself a strong evidence of relativistic bulk motion: the short duration and the even shorter variability timescale (of the order of $1 \mathrm{~ms}$ ) imply a huge compactness (i.e. the luminosity over size ratio). This resembles the conditions during the Big Bang, and implies a similar evolution: no matter in which form the energy is initially injected, a quasi-thermal equilibrium between matter and radiation is reached, with the formation of electron-positron pairs accelerated to relativistic speeds by the high internal pressure. This is a fireball. When the temperature of the radiation (as measured in the comoving frame) drops below $\sim 50 \mathrm{keV}$ the pairs annihilate faster then the rate at which are produced (50 $\mathrm{keV}$, not $511 \mathrm{keV}$, as a thermal photon distribution has a high energy tail...). But the presence of even a small amount of barions, corresponding to only $\sim 10^{-6} M_{\odot}$, makes the fireball opaque to Thomson scattering: the internal radiation thus continues to accelerate the fireball until most of its initial energy has been converted into bulk motion. The fireball then expands at a constant speed and at some point becomes transparent. If the central engine is not completely impulsive, but works intermittently, it can produce many shells (i.e. many fireballs) with slightly different Lorentz factors. Late but faster shells can catch up early slower ones, producing shocks which give rise to the observed burst emission. In the meantime, all shells interact with the interstellar medium, and at some point the amount of swept up matter is large enough to decelerate the fireball and produce other radiation which can be identified with the afterglow emission observed at all frequencies.

For GRBs the limits to the required bulk Lorentz factors follow mainly from two arguments (for reviews see Piran 1999; Meszaros 1999):

- Compactness — We see high energy (i.e. $\gamma$-rays above $100 \mathrm{MeV}$ ) emission, varying on 
short timescales. As for the AGN case (see above) bulk motion is required to lower the implied luminosity and increase the intrinsic size of the emission region in order to decrease the opacity to pair production. Limits to the Lorentz factor in the range 100-300 are derived.

- Variability - The radiation we see originates when the fireball has become optically thin to Thomson scattering, i.e. when it has expanded to radii of the order of $R_{t}=10^{13} \mathrm{~cm}$. Yet, we see $1 \mathrm{~ms}$ variability timescales. Assuming that the radiation is produced while the fireball expands a factor two in radius, short timescales are possible if $\Delta t_{a}=(1-\beta \cos \theta) R_{t} / c$. Furthermore, if isotropic, we always see the portion of it pointing at us $\left(\theta=0^{\circ}\right)$, and thus $\Delta t_{a} \sim 0.01\left(R_{t} / 10^{13}\right)(100 / \Gamma)^{2}$ Also this argument leads to values of $\Gamma$ in the range $100-300$.

\section{$5 \quad$ Rulers and clocks vs photographs and light curves}

In special relativity we are used to two fundamentals effects:

- Lengths shrink in the direction of motion

- Times get longer

The Lorentz transformations for a motion along the $x$ axis are ( $K$ is the lab frame and $K^{\prime}$ is the comoving one)

$$
\begin{aligned}
x^{\prime} & =\Gamma(x-v t) \\
t^{\prime} & =\Gamma\left(1-\frac{v}{c^{2}} x\right)
\end{aligned}
$$

with the inverse relations given by

$$
\begin{aligned}
x & =\Gamma\left(x^{\prime}+v t^{\prime}\right) \\
t & =\Gamma\left(t^{\prime}+\frac{v}{c^{2}} x^{\prime}\right) .
\end{aligned}
$$

The length of a moving ruler has to be measured through the position of its extremes at the same time $t$. Therefore, as $\Delta t=0$, we have

$$
x_{2}^{\prime}-x_{1}^{\prime}=\Gamma\left(x_{2}-x_{1}\right)-\Gamma v \Delta t=\Gamma\left(x_{2}-x_{1}\right)
$$

i.e.

$$
\Delta x=\frac{\Delta x^{\prime}}{\Gamma} \rightarrow \text { contraction }
$$

Similarly in order to determine a time interval a (lab) clock has to be compared with one in the comoving frame, which has, in this frame, the same position $x^{\prime}$. It follows

$$
\Delta t=\Gamma \Delta t^{\prime}+\Gamma \frac{v}{c^{2}} \Delta x^{\prime}=\Gamma \Delta t^{\prime} \rightarrow \text { dilation }
$$

An easy way to remember the transformations is to think to mesons produced in collisions of cosmic rays in the high atmosphere, which can be detected even is their lifetime (in the comoving frame) is much shorter than the time needed to reach the earth's surface. For us, on ground, relativistic mesons live longer.

All this is correct if we measure lengths by comparing rulers (at the same time) and by comparing clocks (at the same position) - the meson lifetime $i s$ a clock. In other words, if we do not use photons for the measurement process. 


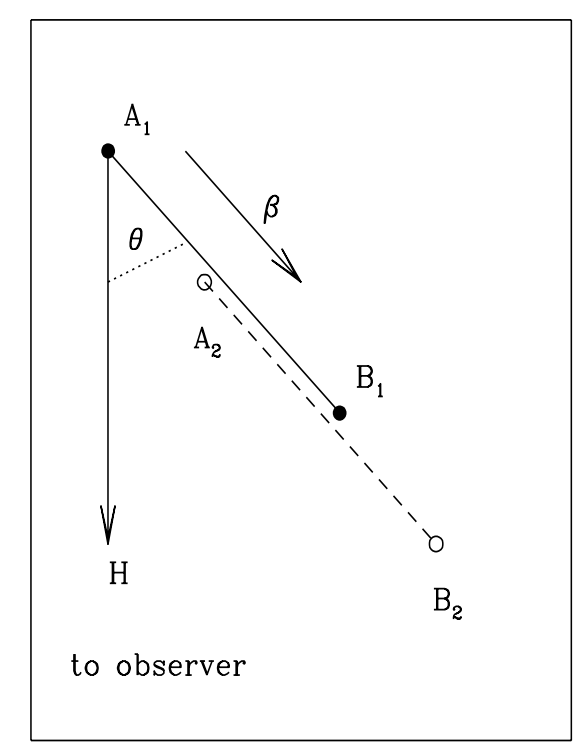

Figure 2: A bar moving with velocity $\beta c$ in the direction of its length. The path of the photons emitted by the extreme $A$ is longer than the path of photons emitted by $B$. When we make a picture of the bar (or a map), we collect photons reaching the detector simultaneously . Therefore the photons from $A$ have to be emitted before those from $B$, when the bar occupied another position.

\subsection{The moving bar}

If the information (about position and time) are carried by photons, we must take into account their (different) paths. When we take a picture, we detect photons arriving at the same time to our camera: if the moving body which emitted them is extended, we must consider that these photons have been emitted at different times, when the moving object occupied different locations in space. This may seem quite obvious. And it is. Nevertheless these facts were pointed out in 1959 (Terrel 1959), more than 50 years after the publication of the theory of special relativity.

Let us consider a moving bar, of proper dimension $\ell^{\prime}$, moving in the direction of its length at velocity $\beta c$ and at an angle $\theta$ with respect to the line of sight (see Fig. 2). The length of the bar in the frame $K$ (according to relativity "without photons") is $\ell=\ell^{\prime} / \Gamma$. The photon emitted in $A_{1}$ reaches the point $H$ in the time interval $\Delta t_{e}$. After $\Delta t_{e}$ the extreme $B_{1}$ has reached the position $B_{2}$, and by this time, photons emitted by the other extreme of the bar can reach the observer simultaneously with the photons emitted by $A_{1}$, since the travel paths are equal. The length $B_{1} B_{2}=\beta c \Delta t_{e}$, while $A_{1} H=c \Delta t_{e}$. Therefore

$$
A_{1} H=A_{1} B_{2} \cos \theta \rightarrow \Delta t_{e}=\frac{\ell^{\prime} \cos \theta}{\Gamma(1-\beta \cos \theta)} .
$$

Note the appearance of the term $\delta=1 /[\Gamma(1-\beta \cos \theta)]$ in the transformation: this accounts for both the relativistic length contraction $(1 / \Gamma)$, and the Doppler effect $[1 /(1-\beta \cos \theta)]$. The length $A_{1} B_{2}$ is then given by

$$
A_{1} B_{2}=\frac{A_{1} H}{\cos \theta}=\frac{\ell^{\prime}}{\Gamma(1-\beta \cos \theta)}=\delta \ell^{\prime}
$$



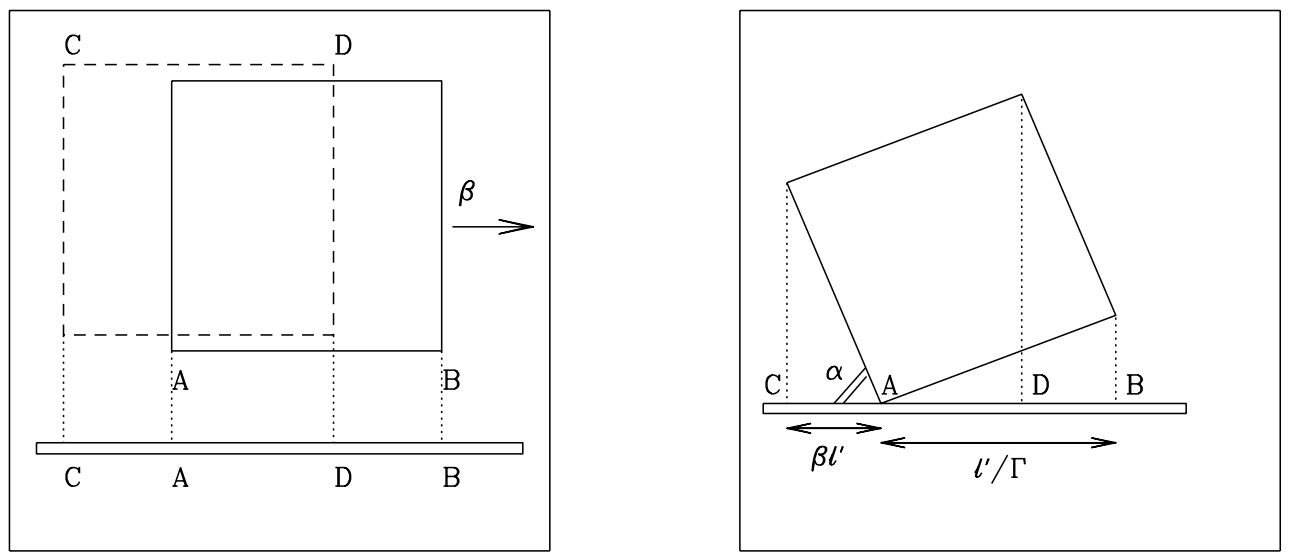

Figure 3: Left: A square moving with velocity $\beta c$ seen at $90^{\circ}$. The observer can see the left side (segment $C A$ ). Light rays are assumed to be parallel, i.e. the square is assumed to be at large distance from the observer. Right: The moving square is seen as rotated by an angle $\alpha$ given by $\cos \alpha=\beta$.

In a real picture, we would see the projection of $A_{1} B_{2}$, i.e.:

$$
H B_{2}=A_{1} B_{2} \sin \theta=\ell^{\prime} \frac{\sin \theta}{\Gamma(1-\beta \cos \theta)}=\ell^{\prime} \delta \sin \theta,
$$

The maximum observed length is $\ell^{\prime}$ for $\cos \theta=\beta$.

\subsection{The moving square}

Now consider a square of size $\ell^{\prime}$ in the comoving frame, moving at $90^{\circ}$ to the line of sight (Fig. $3)$. Photons emitted in $A, B, C$ and $D$ have to arrive to the film plate at the same time. But the paths of photons from $C$ and $D$ are longer $\rightarrow$ they have to be emitted earlier than photons from $A$ and $B$ : when photons from $C$ and $D$ were emitted, the square was in another position. The interval of time between emission from $C$ and from $A$ is $\ell^{\prime} / c$. During this time the square moves by $\beta \ell^{\prime}$, i.e. the length $C A$. Photons from $A$ and $B$ are emitted and received at the same time and therefore $A B=\ell^{\prime} / \Gamma$. The total observed length is given by

$$
C B=C A+A B=\frac{\ell^{\prime}}{\Gamma}(1+\Gamma \beta)
$$

As $\beta$ increases, the observer sees the side $A B$ increasingly shortened by the Lorentz contraction, but at the same time the length of the side $C A$ increases. The maximum total length is observed for $\beta=1 / \sqrt{2}$, corresponding to $\Gamma=\sqrt{2}$ and to $C B=\ell^{\prime} \sqrt{2}$, i.e. equal to the diagonal of the square. Note that we have considered the square (and the bar in the previous section) to be at large distances from the observer, so that the emitted light rays are all parallel. If the object is near to the observer, we must take into account that different points of one side of the square (e.g. the side $A B$ in Fig. 3) have different travel paths to reach the observer, producing additional distortions. See Mook and Vargish (1987) for some interesting illustrations. 


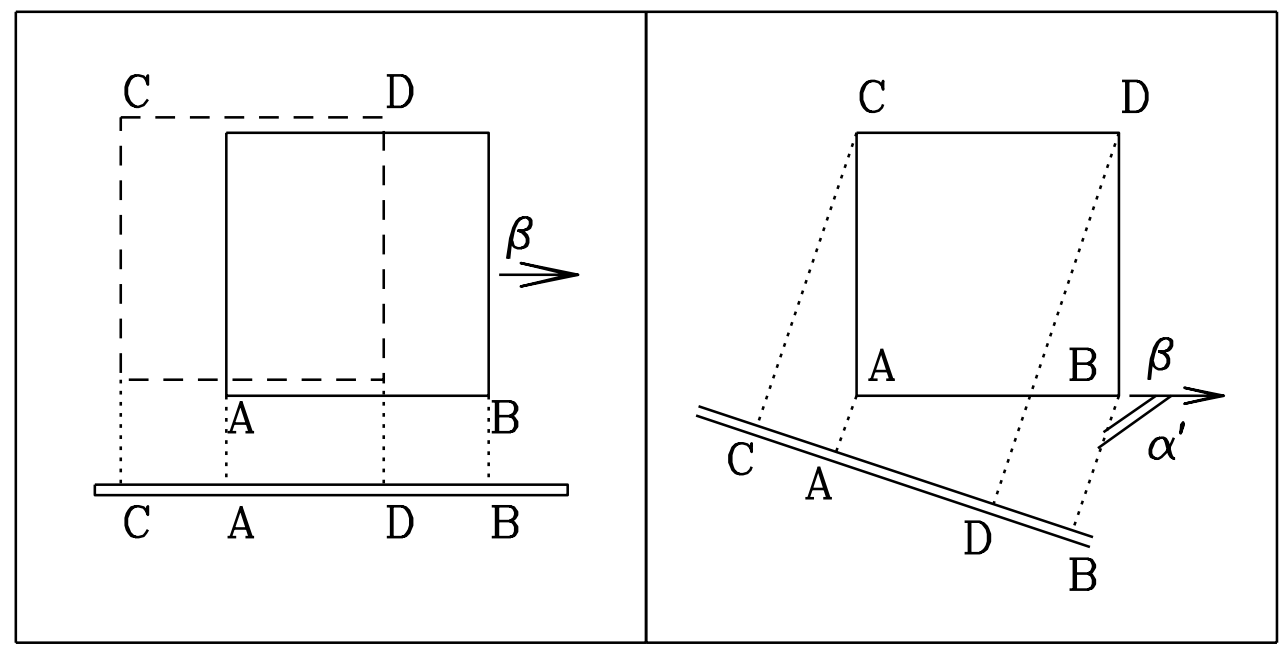

Figure 4: An observer that sees the object at rest at a viewing angle given by $\sin \alpha^{\prime}=\delta \sin \alpha$, will take the same picture as the observer that sees the object moving and making an angle $\alpha$ with his/her line of sight. Note that $\sin \alpha^{\prime}=\sin \left(2 \pi-\alpha^{\prime}\right)$.

\subsection{Rotation, not contraction}

The net result (taking into account both the length contraction and the different paths) is an apparent rotation of the square, as shown in Fig. 3 (right panel). The rotation angle $\alpha$ can be simply derived (even geometrically) and is given by

$$
\cos \alpha=\beta
$$

A few considerations follow:

- If you rotate a sphere you still get a sphere: you do not observe a contracted sphere.

- The total length of the projected square, appearing on the film, is $\ell^{\prime}(\beta+1 / \Gamma)$. It is maximum when the "rotation angle" $\alpha=45^{\circ} \rightarrow \beta=1 / \sqrt{2} \rightarrow \Gamma=\sqrt{2}$. This corresponds to the diagonal.

- The appearance of the square is the same as what seen in a comoving frame for a line of sight making an angle $\alpha^{\prime}$ with respect to the velocity vector, where $\alpha^{\prime}$ is the aberrated angle given by

$$
\sin \alpha^{\prime}=\frac{\sin \alpha}{\Gamma(1-\beta \cos \alpha)}=\delta \sin \alpha
$$

See Fig. 4 for a schematic illustration.

The last point is particularly important, because it introduces a great simplification in calculating not only the appearance of bodies with a complex shape but also the light curves of varying objects.

\subsection{Light curves}

We have already seen how intrinsic time intervals $\Delta t_{e}^{\prime}$ transform in observed $\Delta t_{a}$ when taking into account different photon travel paths. The Doppler effect can oppose to time expansion 
and, depending on the viewing angle, $\Delta t_{e}$ can be longer or shorter than $\Delta t_{e}^{\prime}$. There are however more complex cases, where it may be difficult to derive a prescription as simple as Equation (2). For instance, a relativistically moving blob which also expands relativistically (i.e. "a bomb" exploding in flight). Accounting for the superposition of the two motions is complex, but the introduction of the "aberrated angle observer" greatly simplifies this kind of problems: this observer would see the blob without bulk motion and the different travel paths are the geometric ones. Then the observer in the lab-frame $K$ just sees the same light curve as the "aberrated angle observer", but with time intervals divided by $\delta$ and specific intensities multiplied by $\delta^{3}$. In fact, in the frame $K^{\prime}$ the photons emitted at the "de-aberrated angle" $\alpha^{\prime}$ are the very same ones that reach the observer in $K$, at the "aberrated angle" $\alpha$.

Table 1: Useful transformation

\begin{tabular}{ll}
\hline \hline & frequency \\
$\nu=\delta \nu^{\prime}$ & time \\
$t=t^{\prime} / \delta$ & volume \\
$V=\delta V^{\prime}$ & sine \\
$\sin \theta=\sin \theta^{\prime} / \delta$ & cosine \\
$\cos \theta=\left(\cos \theta^{\prime}+\beta\right) /\left(1+\beta \cos \theta^{\prime}\right)$ & specific intensity \\
$I(\nu)=\delta^{3} I^{\prime}\left(\nu^{\prime}\right)$ & total intensity \\
$I=\delta^{4} I^{\prime}$ & specific emissivity \\
$j(\nu)=\delta^{2} j^{\prime}\left(\nu^{\prime}\right)$ & absorption coefficient \\
$\kappa(\nu)=\kappa^{\prime}\left(\nu^{\prime}\right) / \delta$ & brightness temperature (size directly measured) \\
$T_{B}=\delta T_{B}^{\prime}$ & brightness temperature (size from variability) \\
$T_{B}=\delta^{3} T_{B}^{\prime}$ & \\
\hline \hline
\end{tabular}

\section{Conclusions}

In the last 25 years special relativity has become necessary for the understanding of some of the most violent phenomena in our universe, in which large masses are accelerated to relativistic velocities. Instead of dealing with elementary particles in accelerators or with energetic cosmic rays, we can see fractions of a solar mass moving at $0.999 c$. These motions give raise to several effects, the most important being probably the collimation of the radiation along the direction of motion, making the source visible to distant observers. This implies that relativistic emitting sources - such as the plasma in jets of AGNs and gamma-ray bursts - can be good probes of the far universe.

As discussed in this contribution, spatial and temporal information are carried by photons, and therefore the differences in their paths to reach the observer must be taken into account. Extended moving objects are seen rotated and therefore spheres remain spheres.

\section{Acknowledgments}

It is a pleasure to thank Annalisa Celotti, Laura Maraschi, Aldo Treves and Meg Urry for years of fruitful collaboration. 


\section{References}

[1] Blandford R.D. \& Rees M.J., 1987, in Pittsburgh Conference on BL Lac objects, ed. A.N. Wolfe (Pittsburgh, Univ. of Pittsburgh Press), p. 161.

[2] Garrington S.T., Leahy J.P., Conway R.G. \& Laing R.A., 1988, Nature, 331, 147

[3] Ghisellini G., Padovani P., Celotti A. \& Maraschi L., 1993, ApJ, 407, 65

[4] Gliozzi M., Bodo G. \& Ghisellini G., 1999, MNRAS, 303, L37

[5] Hjellming R.M. \& Rupen M.P., 1995, Nature, 375, 464

[6] Hoyle F.S.R., Burbidge G.R. \& Sargent W.L.W., 1966, Nature, 209, 751

[7] Laing R.A., 1988, Nature, 331, 149

[8] Meszaros P., 1999, Nuclear Phys. B, (Proceedings Supplements), Elsevier Science, in press (astro-ph/9904038)

[9] Mirabel I.F. \& Rodriguez L.F., 1994, Nature, 371, 46

[10] Mook D.E. \& Vargish T., 1987, Inside Relativity, Princeton Univ. Press (Princeton)

[11] Piran T., 1999, Physics Reports, in press astro-ph/9810256

[12] Readhead A.C.S., 1994, ApJ, 426, 51

[13] Rees M.J., 1966, Nature, 211, 468

[14] Rybicki G.B. \& Lightman A. P., 1979, Radiative processes in astrophysics, John Wiley \& Sons (New York)

[15] Terrel J., 1959 , Phys. Rev., 116, 1041

[16] Urry M.C. \& Padovani P., 1995, PASP, 107, 803

[17] Vermeulen R.C. \& Cohen M.H., 1994, ApJ, 430, 467

[18] Wagner S.J. \& Witzel A., 1995, ARA\&A, 33, 163 